\title{
Amyloid precursor protein as a potential marker of malignancy and prognosis in papillary thyroid carcinoma
}

\author{
ZHILI YANG, YOUBEN FAN, ZHAOXIAN DENG, BO WU and QI ZHENG \\ Department of Surgery, The Sixth People's Hospital Affiliated to Shanghai Jiao Tong University, \\ Shanghai 200233, P.R. China
}

Received December 28, 2011; Accepted February 22, 2012

DOI: 10.3892/ol.2012.639

\begin{abstract}
Papillary thyroid carcinoma (PTC) is the most common carcinoma of the thyroid gland and has a relatively favorable prognosis. However, it is important to identify PTC characteristics that indicate a high risk for recurrence and metastasis. Recent data indicate that the amyloid precursor protein (APP) is involved in cell adhesion, motility and proliferation. At present, the expression levels of APP and their prognostic significance in PTC have not been studied. In the present study, the APP gene expression in 10 PTCs and their adjacent thyroid tissue samples was analyzed using quantitative real time-PCR. Tissue array blocks were obtained from 90 PTC cases. Tumor regions and their adjacent non-tumor regions were analyzed in tissue array blocks and immunohistochemistry was conducted using sectioned slides. Semi-quantitative scores were correlated with the clinicopathological factors of 90 PTC patients. APP-specific mRNA was increased 50-fold in PTC samples compared with their adjacent thyroid tissue. The immunohistochemistry results showed APP expression levels to be significantly increased in PTC samples compared with their adjacent non-tumor thyroid tissue $(\mathrm{p}<0.001)$. High APP scores were significantly correlated with large tumor size, extracapsular invasion and lymph node metastasis. The amyloid precursor protein was overexpressed in PTC and a high APP expression was associated with high malignant potential. Therefore, APP may serve as a prognostic marker and potential novel therapeutic target in PTC.
\end{abstract}

\section{Introduction}

Papillary thyroid cancer (PTC) is the most common welldifferentiated cancer of the thyroid. It represents approximately $80-85 \%$ of well-differentiated thyroid cancers (1). However, some cases show relatively early recurrence, severe invasion,

Correspondence to: Professor Qi Zheng, Department of Surgery, The Sixth People's Hospital Affiliated to Shanghai Jiao Tong University, 600 Yishan Road, Shanghai 200233, P.R. China

E-mail: zhengqi1957@yahoo.com.cn

Key words: amyloid precursor protein, papillary thyroid carcinoma, prognosis multiple lymph node metastasis or distant metastasis (2). Therefore, it is important to identify those characteristics of PTC that have a high risk for invasion and metastasis.

The amyloid precursor protein (APP) is a transmembrane protein that contains a large $\mathrm{N}$-terminal ectodomain and a short C-terminal endodomain and is expressed in the central nervous system and peripheral tissues, including epidermis, pancreas and thyroid (3-5). A proteomic study revealed an increased expression of APP in benign cold thyroid nodules compared with normal thyroid tissue (6). In a previous study, the upregulation of APP expression was induced by TSH in differentiated thyroid cells and by insulin in thyroid cancer cells in vitro; thyroid cancers are characterized by APP upregulation, increased membrane targeting of the APP ectodomain and significantly increased mRNA levels of the APP scaffold proteins (7). However, correlations between APP expression and clinicopathological parameters have yet to be investigated in PTC.

The present study aimed to investigate the expression levels of the APP in PTC and to examine clinicopathological correlations such as invasive and metastatic characteristics.

\section{Materials and methods}

Case selection. Tumor specimens for quantitative real timePCR were obtained from 10 PTC patients who underwent total thyroidectomy at the Department of Surgery, Shanghai Jiao Tong University Affiliated Sixth People's Hospital, China. Non-tumor thyroid tissues $(n=10)$ were obtained from tissue adjacent to each PTC exhibiting apparently non-tumor morphology as a control.

Tissues for immunohistochemistry were randomly selected from 90 PTC patients who underwent surgery in the same department between 2008 and 2010. Non-tumor thyroid tissues $(n=90)$ were obtained from adjacent tissue of PTC patients exhibiting apparently non-tumor morphology as a control. These patients included 20 males and 70 females, and the average age was 45.2 years. The study was approved by the Ethics Committee of the Sixth People's Hospital Affiliated to Shanghai Jiao Tong University.

Tissue sample preparation and tissue microarray. Following surgical resection, specimens from the 90 PTC patients were fixed with $10 \%$ formalin. Sections of PTC tissue cores were 
stained with hematoxylin and eosin to identify areas of tumor and non-tumor tissue. When the areas of interest were identified, the recipient tissue array block was constructed using manual tissue array equipment (IHC World, Woodstock, MD, USA). Cores (2-mm) were placed in the recipient block, which was heated to fix the samples and a paraffin layer was applied to ensure proper facing. To facilitate blind grading, an Excel spreadsheet (Microsoft Corporation, Redmond, WA, USA) was constructed using sample accession numbers but without information regarding the final pathological finding.

Immunohistochemistry. Sectioned slides were deparaffinized three times in xylene for 20 min each and rehydrated using a graded alcohol solution. Antigen retrieval was performed in citrate buffer in a microwave. Endogenous peroxidase activity was quenched by incubating in peroxidase-blocking reagent. Sections were incubated in the primary antibody of APP (22C11, 1:50, Millipore, Billerica, MA, USA). The second antibody was subsequently incubated for $30 \mathrm{~min}$ and counterstained with hematoxylin.

Quantitative real-time-PCR. Total RNA was prepared from tissues using the RNeasy extraction kit (GE Healthcare, Amersham, UK) and reverse transcribed using high-capacity cDNA reverse transcription kits (Applied Biosystems, Melbourne, Australia) according to the manufacturer's instructions. Quantitative real-time PCR (qRT-PCR) was performed on a 7300 Fast Real-Time PCR System (Applied Biosystems) using SYBR-Green PCR Master mix (Applied Biosystems). The human-specific intron spanning primer pairs for APP were: forward: 5'-CCGATGATGACGAGGACGAT-3' and reverse: 5'-GTGGTGGTGGTGGCAATG-3'. The primer pairs used for GAPDH were: forward: 5'-CAATGACCCCTTCATTGACC-3' and reverse: 5'-TGATGACAAGCTTCCCGTTC-3'. Cycle conditions were as follows: 1 cycle at $50^{\circ} \mathrm{C}$ for $2 \mathrm{~min}$, followed by 1 cycle at $95^{\circ} \mathrm{C}$ for $10 \mathrm{~min}, 40$ cycles at $95^{\circ} \mathrm{C}$ for $15 \mathrm{sec}$ and $60^{\circ} \mathrm{C}$ for $1 \mathrm{~min}$. Specificity of PCR products was tested by dissociation curves. Relative values of transcripts were calculated using the equation, $2^{-\Delta \Delta \mathrm{Ct}}$, where $\Delta \mathrm{Ct}$ is equal to the difference in threshold cycles for the target and reference. Each experiment was performed in triplicate.

Immunohistological scores and clinicopathological parameters. A surgical pathologist blinded to the identity of the specimens examined the percentage of positivity and intensity of immunostained slides and scored them as previously described (8). The percentage of positivity was the number of cells showing positive staining (grade 0, 0; grade 1, 1-33\%; grade 2, 34-66\%; and grade 3,67-100\%, respectively). The intensity grade 0 was defined as no immunoreaction, grade 1 as weak immunoreaction, grade 2 as moderate immunoreaction, and grade 3 as strong immunoreaction. The total scores in PTC (tumor score) and non-tumor tissues (non-tumor score) were determined as the sum of the positivity and intensity grades.

Based on the clinical and pathological record, a retrospective analysis was performed on the following variables: age, gender, tumor size, lymph node metastasis, extracapsular invasion, multifocality, distant metastasis, and clinical stage. Clinical stage was determined according to the pathological
Table I. Immunohistochemical analysis of APP in tumor regions and non-tumor regions from the same thyroidectomy specimens according to the scoring system and positive immunoreactivity.

\begin{tabular}{|c|c|c|c|}
\hline & $\mathrm{n}$ & $\begin{array}{l}\text { Tissues with positive } \\
\text { immunoreactivity }(\%)\end{array}$ & P-value \\
\hline Tumor score & & $100(100)$ & $<0.001$ \\
\hline 0 & 0 & & \\
\hline $1-2$ & 10 & & \\
\hline $3-4$ & 35 & & \\
\hline $5-6$ & 45 & & \\
\hline Non-tumor score & & $36(40)$ & \\
\hline 0 & 54 & & \\
\hline $1-2$ & 25 & & \\
\hline $3-4$ & 11 & & \\
\hline $5-6$ & 0 & & \\
\hline
\end{tabular}

The immunohistochemical scores in PTC and non-tumor thyroid tissues were determined as the sum of the positivity and intensity grades. The percentage of positivity was scored from 0 to 4 (grade 0 , $0 \%$; grade $1,1-33 \%$; grade $2,34-66 \%$; and grade $3,67-100 \%$ ). The intensity grade was scored from 0 to 4 (grade 0 , no immunoreactions; grade 1, weak immunoreactions; grade 2, moderate immunoreactions; grade 3, strong immunoreactions). Positive immunoreactivity was defined as the immunohistochemical score $>0$ in tumor and non-tumor regions, respectively.

TNM system (NCCN Guidelines, Thyroid Carcinoma, Version 2.2011). Immunohistochemical results were correlated with the clinicopathological parameters to evaluate the prognostic significance.

Statistical analysis. Scores were shown as the mean \pm standard deviation. Statistical analysis was performed using SPSS statistical software (version 17.0; SSPS, Chicago, IL, USA). The Mann-Whitney U test was used to compare the expression levels of the APP gene by quantitative PCR between PTC and non-tumor tissue. The independent samples t-test was used to compare average scores of tumor marker using immunohistochemistry and clinicopathological variables. The number of positive immunoreactions (tumor score $>0$ ) in PTC and normal thyroid tissues were evaluated using the Chi-square test. $\mathrm{P}<0.05$ was accepted as indicative of a significant difference.

\section{Results}

Expression levels of APP mRNA in PTC and non-tumor thyroid tissues. To compare the gene expression in PTC and non-tumor thyroid tissues, APP mRNA expression was analyzed by quantitative real time-PCR. APP mRNA increased approximately 50-fold in PTC compared with nontumor thyroid tissues (Fig. 1).

Immunohistochemical expression of APP in PTC and nontumor thyroid tissues. Tissue microarrays were constructed to 


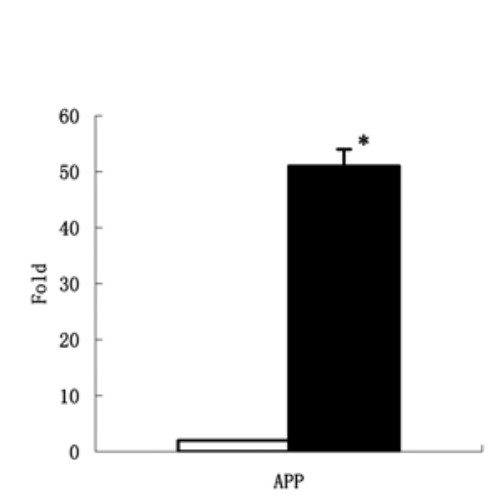

Qnon-tumor tissue
QPTC

Figure 1. qRT-PCR assay of relative APP mRNA levels in PTC and nontumor thyroid tissues. Data were normalized to the expression of the housekeeping gene glyceraldehyde-3-phosphate dehydrogenase. The y-axis shows an increase in specific mRNA over unstimulated samples. Data are the mean \pm standard deviation from triplicate experiments. ${ }^{*} \mathrm{P}<0.001$ compared with non-tumor tissues.

investigate the differences in APP protein expression between PTC and non-tumor thyroid tissues (Fig. 2).

In the tumor regions, positive immunoreactions for APP (tumor score $>0$ ) were present in 90 of 90 cases $(100 \%)$. In the non-tumor regions, positive immunoreactions for APP (nontumor score $>0$ ) were present in 36 of 90 cases $(40 \%)$. APP protein expression levels were increased in the tumor regions compared to the non-tumor regions $(\mathrm{P}<0.001$; Table I).

Correlation between the immunohistochemical scores and clinicopathological parameters. APP high scores significantly correlated with and clinicopathological data such as large tumor size, extracapsular invasion, and lymph node metastasis (Table II).
Table II. Prevalence of APP tumor scores and clinicopathological parameters.

\begin{tabular}{lccc}
\hline & $\mathrm{n}(\%)$ & APP score & P-value \\
\hline Age (years) & & & \\
$<45$ & $50(55)$ & $3.25 \pm 2.01$ & NS \\
$\geq 45$ & $40(45)$ & $3.30 \pm 1.90$ & \\
Gender & & & \\
$\quad$ Male & $20(20)$ & $3.78 \pm 1.98$ & NS \\
$\quad$ Female & $70(80)$ & $3.77 \pm 1.85$ & \\
Size (cm) & & & \\
$\quad \leq 2$ & $54(60)$ & $2.82 \pm 2.30$ & 0.005 \\
$>2$ & $36(40)$ & $4.52 \pm 2.12$ & \\
Extracapsular invasion & & & \\
$\quad$ No & $63(70)$ & $3.58 \pm 1.45$ & 0.024 \\
Yes & $27(30)$ & $4.64 \pm 2.50$ & \\
Multifocality & & & \\
$\quad$ No & $75(83)$ & $3.79 \pm 2.45$ & NS \\
Yes & $15(17)$ & $3.84 \pm 1.45$ & \\
LN metastasis & & & \\
No & $68(75)$ & $2.98 \pm 2.46$ & 0.004 \\
$\quad$ Yes & $22(25)$ & $4.95 \pm 1.55$ & \\
Distant metastasis & & & \\
$\quad$ No & $81(90)$ & $3.74 \pm 2.21$ & NS \\
$\quad$ Yes & $9(10)$ & $3.80 \pm 2.25$ & \\
TNM stage & & & \\
I and II & $72(80)$ & $3.71 \pm 2.25$ & NS \\
III and IV & $18(20)$ & $3.74 \pm 1.91$ & \\
\hline
\end{tabular}

Tumor scores were determined as the sum of the positivity and intensity grades in the tumor region. LN, lymph node. NS, not significant.
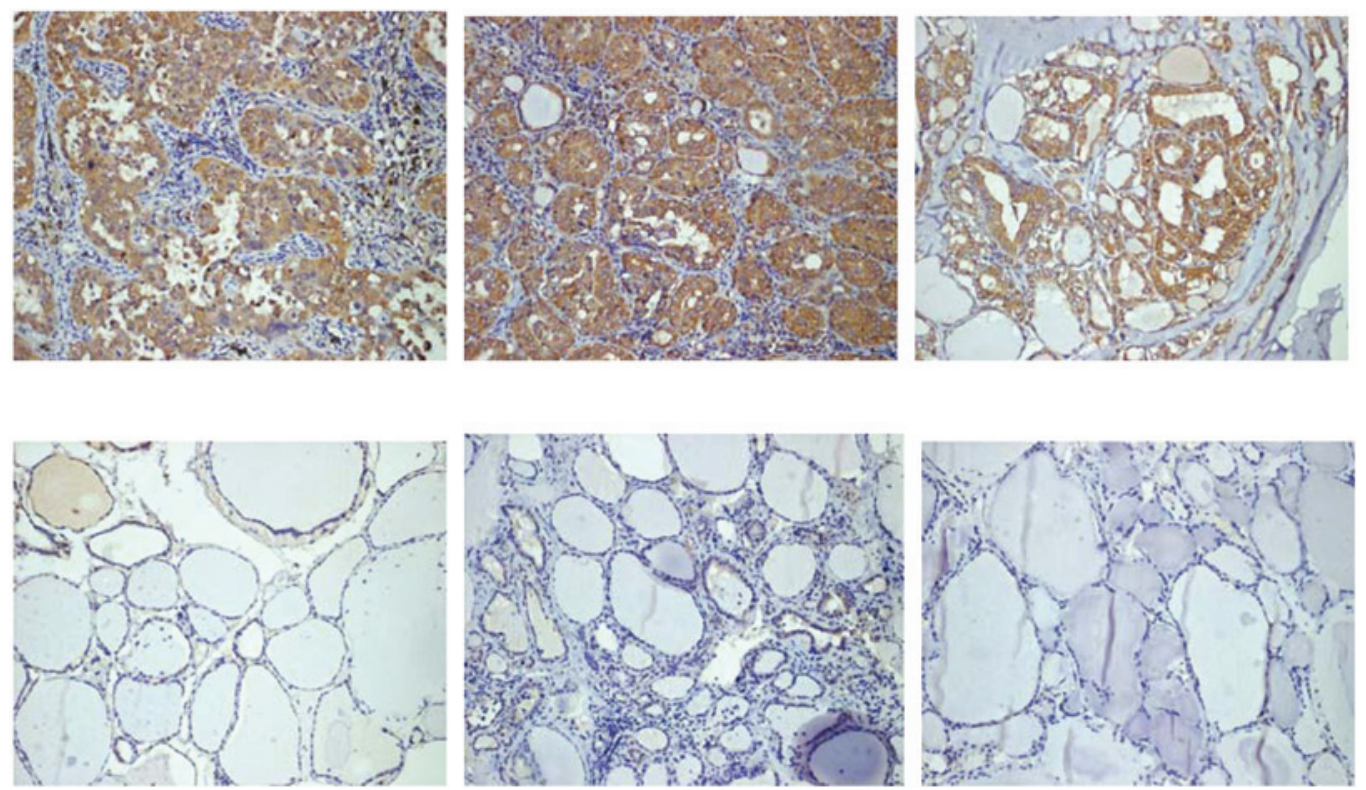

Figure 2. Representative images of APP expression in (A) three papillary thyroid carcinoma samples and (B) their adjacent non-tumor tissue samples (magnification, x200). 


\section{Discussion}

APP undergoes several types of proteolytic processing, one of which results in the release of the secretory $\mathrm{N}$-terminal portion of APP (sAPP), which carries a number of biologically relevant domains. The ability of APP to induce growth-promoting effects and morphological alterations occurs not only through effects of the sAPP but may also occur through effects of the cytosolic domain of APP, whether cleaved or intact. The presence of multiple cytosolic interactors of APP has been identified, including JIP1b, Fe65, ShcA and APPBP1, many of which may function in a neoplastic system to induce cell proliferation and migration (5).

Overexpression of APP was observed in other human cancer types, such as prostate (9), colon (10), parathyroid (11), pancreatic (5), and oral cancers(12). The present study showed that APP expression was upregulated in PTC compared with non-tumor thyroid tissues at the transcription and protein levels. APP acts at the cell surface to bind and activate signaling proteins via its intracellular domain (AICD); Fe65, an AICD-binding protein, has been shown to stimulate APP trafficking and APP proteolysis, which in turn leads to increased sAPP secretion (13). Combined with the results of the present study, this finding indicates that a high expression of the APP in PTC may increase sAPP secretion. Thyrotropin (TSH) reportedly induced APP expression and sAPP release in differentiated thyroid cells (FRTL-5), and promoted change from differentiation to proliferation in thyroid epithelial cells (14), indicating that sAPP operates as an autocrine growth factor mediating the proliferative effect of TSH on neighboring thyroid epithelial cells. Therefore, the present results indicate that APP expression may be associated with thyroid carcinogenesis and be of use as a diagnostic marker.

Findings of certain studies have shown that APP and its secreted forms promote adhesion, migration, neurite outgrowth, and general growth-promoting properties (15). Therefore, we investigated the correlation between the expression levels of APP and clinicopathological parameters such as invasive and metastatic characteristics in PTC. Our results showed that high APP scores significantly correlated with large tumor size, extracapsular invasion, and lymph node metastasis in PTC. Several studies have shown that patients with increased APP levels have a significantly lower survival rate and APP has therefore been suggested as a potential biomarker for the evaluation of cancer prognosis $(5,9,10,12)$. For PTC patients, tumor size, lymph node metastasis and extracapsular invasion are recognized as important prognostic factors (16). Therefore, the results of the present study indicate that a high APP expression may be correlated with poor prognosis in patients with PTC.
In conclusion, our results confirm previous observations that APP is a crucial mediator of tumor growth in general, and provides evidence of a role for APP in PTC diagnosis and prognosis. APP may therefore provide a molecular target for the therapeutic intervention of PTC. More studies regarding the role of APP and intracellular signaling pathways in PTC are required.

\section{References}

1. Davies L and Welch HG: Increasing incidence of thyroid cancer in the United States, 1973-2002. JAMA 295: 2164-2167, 2006.

2. Hay ID, Thompson GB, Grant CS, et al: Papillary thyroid carcinoma managed at the Mayo Clinic during six decades (1940-1999): temporal trends in initial therapy and long-term outcome in 2444 consecutively treated patients. World J Surg 26: 879-885, 2002.

3. Hoffmann J, Twiesselmann C, Kummer MP, Romagnoli P and Herzog V: A possible role for the Alzheimer amyloid precursor protein in the regulation of epidermal basal cell proliferation. Eur J Cell Biol 79: 905-913, 2000.

4. Graebert KS, Lemansky P, Kehle T and Herzog V: Localization and regulated release of Alzheimer amyloid precursor-like protein in thyrocytes. Lab Invest 72: 513-523, 1995.

5. Hansel DE, Rahman A, Wehner S, Herzog V, Yeo CJ and Maitra A: Increased expression and processing of the Alzheimer amyloid precursor protein in pancreatic cancer may influence cellular proliferation. Cancer Res 63: 7032-7037, 2003.

6. Krause K, Karger S, Schierhorn A, Poncin S, Many MC and Fuhrer D: Proteomic profiling of cold thyroid nodules. Endocrinology 148: 1754-1763, 2007.

7. Krause K, Karger S, Sheu SY, et al: Evidence for a role of the amyloid precursor protein in thyroid carcinogenesis. J Endocrinol 198: 291-299, 2008.

8. Nam KH, Noh TW, Chung SH, et al: Expression of the membrane mucins MUC4 and MUC15, potential markers of malignancy and prognosis, in papillary thyroid carcinorma. Thyroid 21: 745-750, 2011.

9. Takayama K, Tsutsumi S, Suzuki T, et al: Amyloid precursor protein is a primary androgen target gene that promotes prostate cancer growth. Cancer Res 69: 137-142, 2009.

10. Meng JY, Kataoka H, Itoh H and Koono M: Amyloid $\beta$ protein precursor is involved in the growth of human colon carcinoma cell in vitro and in vivo. Int J Cancer 92: 31-39, 2001.

11. Haven CJ, Howell VM, Eilers PH, et al: Gene expression of parathyroid tumors: molecular subclassification and identification of the potential malignant phenotype. Cancer Res 64: 7405-7411, 2004.

12. Ko SY, Lin SC, Chang KW, Wong YK, Liu CJ, Chi CW and Liu TY: Increased expression of amyloid precursor protein in oral squamous cell carcinoma. Int J Cancer 111: 727-732, 2004.

13. King GD and Scott Turner R: Adaptor protein interactions: modulators of amyloid precursor protein metabolism and Alzheimer's disease risk? Exp Neurol 185: 208-219, 2004.

14. Pietrzik CU, Hoffmann J, Stöber K, et al: From differentiation to proliferation: the secretory amyloid precursor protein as a local mediator of growth in thyroid epithelial cells. Proc Natl Acad Sci USA 95: 1770-1775, 1998.

15. Thinakaran $G$ and Koo EH: Amyloid precursor protein trafficking, processing, and function. J Biol Chem 283: 29615-29619, 2008.

16. Ito $\mathrm{Y}$ and Miyauchi A: Prognostic factors of papillary and follicular carcinomas in Japan based on data of Kuma hospital. J Thyroid Res 2012: 973497, 2012. 